\title{
Improving teams in healthcare
}

\author{
Authors: Judith Tweedie, ${ }^{A}$ Lewis Peake, ${ }^{B}$ Nina Dutta ${ }^{B}$ and Andrew Goddard ${ }^{B}$
}

\section{Aims}

This project's primary aim was to empower physicians to create and maintain high functioning healthcare teams, in the context of unprecedented low morale and organisational pressures.

\section{Methods}

Three methodologies were employed to address the following objectives:

$>$ to provide evidence-based guidance on creating and maintaining high functioning medical teams

$>$ to raise awareness of the importance of good teamwork and communication in the medical profession.

The three methodologies used follow.

1 A literature search focusing on three thematic areas: a current perceptions of teamwork in healthcare

b facilitators to effective team working

c barriers to effective team working.

2 An analysis of the current healthcare landscape with respect to teamwork, and the identification of key stakeholders. The authors convened a national event, with representatives from stakeholder organisations, where the evidence arising from the literature review was discussed and evaluated. Recommendations for improving teams in healthcare were identified.

3 Collation of case studies and best practice examples from healthcare professionals in practice.

\section{Results}

The data collected by these three methodologies were synthesised and published in four individual but complementary resources. Each publication centred on a different aspect of team working and presented relevant literature and best practice examples. Key action points and take home messages were also presented, to aid with application. The resources have had a fantastic response, with good engagement from clinicians; one piece of feedback referred to them as 'fascinating and insightful', applicable to 'clinicians of all stages, particularly consultants and registrars'.

In total, the publications have been downloaded 846 times (the 13th most downloaded resource, from a potential of 1,714 hosted on the website) since launch.

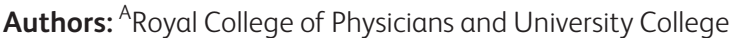

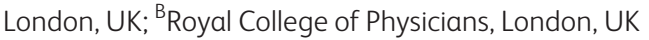

The publications were produced in collaboration with five major national stakeholder groups, verifying the quality and accessibility of the work. In addition, the wider project team has received funding to develop and deliver a second phase building on the momentum from phase one.

\section{Conclusion}

This project has successfully achieved its objectives. It provides physicians with an accessible resource to assist in their team's development, while increasing awareness of the importance of good teamwork in healthcare. The case studies and best practice examples contained in each publication add to the bank of knowledge teams can employ to improve effectiveness and healthcare worker morale.

\section{Conflict of interest statement}

None declared. 